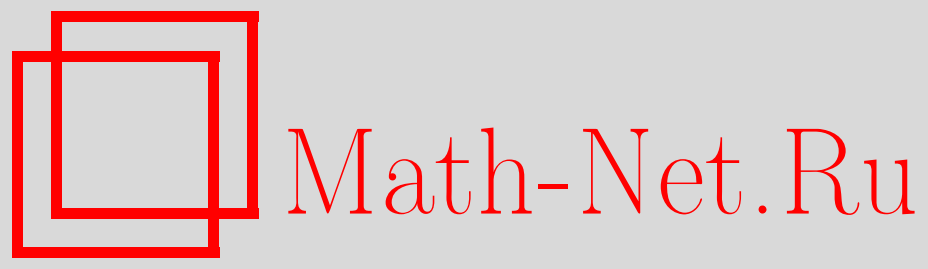

Б. С. Бычков, О разложениях циклической перестановки в произведение данного числа перестановок, Функи. анализ и его прил., 2015, том 49, выпуск $2,1-6$

DOI: https://doi.org/10.4213/faa3187

Использование Общероссийского математического портала Math$\mathrm{Net.Ru}$ подразумевает, что вы прочитали и согласны с пользовательским соглашением http://www. mathnet.ru/rus/agreement

Параметры загрузки:

IP : 3.85 .73 .92

26 апреля 2023 г., $17: 42: 49$

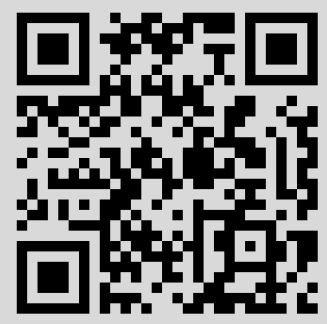




\title{
О разложениях циклической перестановки в произведение данного числа перестановок*
}

\author{
(c) 2015. Б. С. Бычков
}

\begin{abstract}
Исследование разложений перестановки в произведение перестановок, удовлетворяющих некоторым условиям, играет ключевую роль в изучении мероморфных функций или, что то же самое, разветвленных накрытий двумерной сферы; оно восходит к работам А. Гурвица конца XIX в. В 2000 г. М. Буске-Мелу и Ж. Шеффер получили элегантную формулу для количества разложений перестановки в произведение заданного числа перестановок, отвечающих накрытиям рода 0. Обобщения их формулы на накрытия сферы поверхностями старших родов неизвестны до сих пор. В статье дается новое доказательство формулы Буске-Мелу-Шеффера для случая разложений циклической перестановки, которое, как можно надеяться, допускает обобщение на положительные роды.
\end{abstract}

\section{§1. Введение}

Задача перечисления разложений данной перестановки в произведение перестановок восходит к Гурвицу [6]. В этой статье 1891 г. была получена формула для числа разложений рода 0 перестановки фиксированного циклического типа в произведение транспозиций (интересующийся читатель найдет точные формулировки ниже в тексте). Впоследствии эти числа стали называть числами Гурвица. Большой прорыв в этой области был сделан только в начале XXI в.: в работе [3] было получено выражение для числа Гурвица произвольного рода. Наряду с другими, эта работа породила бурный всплеск исследований на указанную тему. За последнее десятилетие появился целый ряд работ по исследованию так называемых двойных чисел Гурвица ([9], [7]), по перечислению разветвленных накрытий двумерной сферы с несколько отличными от данных Гурвица фиксированными данными о критических значениях ([2], [5]) и по многим близким вопросам. В частности, в работе [2] получена формула для количества разложений рода 0 данной перестановки $\sigma_{0}$ фиксированного циклического типа в произведение $c$ перестановок произвольных циклических типов. В настоящей статье получено новое доказательство формулы из [2] в случае, когда перестановка $\sigma_{0}$ является полным циклом. Доказательство основано на формуле Гульдена-Джексона из [4], перечисляющей упорядоченные разложения полного цикла в произведение перестановок данных циклических типов.

Полученные результаты позволяют рассчитывать на то, что подобные рассуждения могут иметь дальнейшие обобщения на случаи разложений положительных родов.

Автор благодарен С. К. Ландо за постановку задачи и постоянное внимание к работе.

* Работа выполнена при поддержке грантов РФФИ 12-01-31233, 13-01-00383 и частичной поддержке фонда Саймонса. 
Ниже мы формулируем результаты, на которые будет опираться доказательство.

1. Формула Гульдена-Джексона. Пусть $\lambda=1^{m_{1}} \cdots n^{m_{n}}$ - разбиение числа $n$ длины $l(\lambda)=m_{1}+\cdots+m_{n}$. Числа $m_{i}$ равны количеству частей разбиения $\lambda$ длины $i$. Будем говорить, что перестановка $\sigma$ из симметрической группы $S_{n}$ имеет циклический тип $\lambda$, если она имеет $m_{i}$ циклов длины $i$. Циклические типы перестановок находятся во взаимно однозначном соответствии с их классами сопряженности.

Пусть $\sigma_{0}$ - полный цикл из $S_{n}$, и пусть $N\left(\lambda_{1}, \ldots, \lambda_{c}\right)$ - число упорядоченных разложений перестановки $\sigma_{0}$ в произведение $c$ перестановок фиксированных циклических типов $\lambda_{1}, \ldots, \lambda_{c}$. В работе [4] получена следующая формула для чисел $N\left(\lambda_{1}, \ldots, \lambda_{c}\right)$ :

Теорема 1.1 (Гульден-Джексон).

$$
N\left(\lambda_{1}, \ldots, \lambda_{c}\right)=n^{c-1} \prod_{i=1}^{c} \frac{1}{l\left(\lambda_{i}\right)} \frac{l\left(\lambda_{i}\right) !}{m_{i 1} ! \cdots m_{i n} !},
$$

где $m_{i j}$ - это количество частей разбиения $\lambda_{i}$ длины $j$.

2. Формула Буске-Мелу-Шеффера. Каждому разложению перестановки из $S_{n}$ в произведение перестановок соответствует разветвленное накрытие сферы степени $n$, определенное однозначно с точностью до топологической эквивалентности. Если группа, порожденная этим набором перестановок, действует транзитивно на множестве из $n$ элементов, то соответствующее накрытие оказывается связным. Род накрывающей поверхности определяется по формуле Римана-Гурвица. Будем говорить, что разложение перестановки в произведение перестановок имеет род $g$, если соответствующее разветвленное накрытие связно и род накрывающей поверхности равен $g$.

Обозначим через $b_{\sigma_{0}}(c)$ количество разложений перестановки $\sigma_{0} \in S_{n}$ фиксированного циклического типа в произведение $c$ перестановок (некоторые из которых могут быть тождественными), удовлетворяющих следующим условиям:

(a) группа, порожденная этим набором из $c$ перестановок, действует транзитивно на множестве из $n$ элементов;

(b) соответствующее разветвленное накрытие имеет род 0 .

В [2] получена следующая формула для чисел $b_{\sigma_{0}}(c)$, которые мы будем называть числами Буске-Мелу-Шеффера.

Теорема 1.2 (Буске-Мелу-Шеффер). Пусть перестановка $\sigma_{0} \in S_{n}$ имеет $m_{i}$ чиклов длины $i(i=1,2, \ldots)$, а $l\left(\sigma_{0}\right)$ обозначает количество ииклов этой перестановки. Тогда

$$
b_{\sigma_{0}}(c)=c \frac{(c n-n-1) !}{\left(c n-n-l\left(\sigma_{0}\right)+2\right) !} \prod_{i \geqslant 1}\left(i\left(\begin{array}{c}
c i-1 \\
i
\end{array}\right)\right)^{m_{i}} .
$$

Через $b_{n}(c)$ обозначим число Буске-Мелу-Шеффера в случае, когда перестановка $\sigma_{0}$ - это полный цикл длины $n$. 
3. Результаты. Вырожденностью $A(\lambda)$ разбиения $\lambda$ называется величина

$$
A(\lambda)=\sum_{i=1}^{n}(i-1) m_{i} .
$$

Вырожденностью перестановки из $S_{n}$ будем называть вырожденность соответствующего разбиения числа $n$.

Зафиксируем натуральное число $c$, и пусть $k_{1}, \ldots, k_{c}$ - невозрастающая последовательность неотрицательных целых чисел, $k_{1} \geqslant \cdots \geqslant k_{c} \geqslant 0, k_{1}+\cdots+k_{c}=$ $n-1$. Рассмотрим наборы из $c$ перестановок вырожденностей $k_{1}, \ldots, k_{c}$, дающих в произведении полный цикл длины $n$. Отметим, что так как мы рассматриваем разложения полного цикла, то группа, порожденная этим набором из $c$ перестановок, действует транзитивно на множестве из $n$ элементов, и поскольку $k_{1}+\cdots+k_{c}=n-1$, из формулы Римана-Гурвица следует, что соответствующее разветвленное накрытие имеет род 0. Таким образом, выполняются условия (a), (b) из разд. 2. Обозначим число таких наборов через $\operatorname{deg}_{k_{1}, \ldots, k_{c}}$.

Лемма 1.3.

$$
\operatorname{deg}_{k_{1}, \ldots, k_{c}}=\frac{1}{n}\left(\begin{array}{c}
n \\
k_{1}
\end{array}\right) \cdots\left(\begin{array}{c}
n \\
k_{c}
\end{array}\right) .
$$

Искомое же число $b_{n}(c)$ равно сумме чисел $\operatorname{deg}_{k_{1}, \ldots, k_{c}}$ по всевозможным наборам вырожденностей $k_{1}, \ldots, k_{c}$, дающих в сумме $n-1$. Отметим, что нулевые вырожденности отвечают появлению тождественных перестановок в разложении Буске-Мелу-Шеффера.

\section{Теорема 1.4 .}

$$
b_{n}(c)=\frac{1}{n}\left(\begin{array}{c}
c n \\
n-1
\end{array}\right) .
$$

Заметим, что обозначения $\operatorname{deg}_{k_{1}, \ldots, k_{c}}$ выбраны не случайно. Эти числа естественно выражаются в терминах степеней ограничения отображения ЛяшкоЛойенги на страты дискриминанта в пространстве комплексных многочленов степени $n$, см. [1]. Более того, все утверждения настоящей статьи могут быть доказаны, опираясь на результаты вычислений степеней отображения ЛяшкоЛойенги из работы [1], однако получающиеся вычисления чуть более громоздки. Именно геометрическая природа выполняемых вычислений позволяет надеяться на то, что излагаемый подход допускает распространение на случай накрытий произвольного рода.

Следующий параграф будет посвящен доказательству основных утверждений, леммы 1.3 и теоремы 1.4 .

\section{§2. Доказательства}

Рассмотрим упорядоченные разложения полного цикла в произведение $c$ перестановок, лишь одна из которых не является транспозицией. Будем называть такую перестановку вырожденной. Пусть $\lambda=1^{m_{1}} \cdots n^{m_{n}}$ - циклический тип единственной вырожденной перестановки. По теореме 1.1 число рассматриваемых разложений равно

$$
N(\lambda)=n^{c-1} \frac{1}{l(\lambda)} \frac{l(\lambda) !}{m_{1} ! \cdots m_{n} !},
$$


поскольку все множители в (1), отвечающие транспозициям, равны 1.

В следующем разделе мы получим формулу для числа упорядоченных разложений полного цикла в произведение $c$ перестановок при условии, что только одна из них является вырожденной и при этом имеет фиксированную вырожденность $k$. Будем обозначать число таких разложений через $\operatorname{deg}_{k}$.

\section{1. Вычисление $\operatorname{deg}_{k}$.}

Утверждение 2.1.

$$
\operatorname{deg}_{k}=\sum_{A(\lambda)=k} N(\lambda)=n^{c-2}\left(\begin{array}{l}
n \\
k
\end{array}\right) .
$$

Представим разбиение $\lambda \vdash n$ в виде диаграммы Юнга площади $n$ (части разбиения записываются по строкам). Отрежем от этой диаграммы первый столбец: получится диаграмма площади $\sum_{i=1}^{n}(i-1) m_{i}$, т. е. в точности площади $k$. Заметим, что количества совпадающих частей у разбиения числа $n$ и у полученного разбиения числа $k$ совпадают, за исключением того, что в разбиении числа $k$ нет частей длины 1 . Поэтому мы будем интерпретировать сумму чисел $N(\lambda)$ по всем разбиениям $\lambda$ числа $n$ вырожденности $k$ как сумму тех же чисел по всем разбиениям $\nu=1^{m_{2}} 2^{m_{3}} \cdots(n-1)^{m_{n}}$ числа $k$ (здесь и далее через $|\operatorname{Aut}(\nu)|$ будем обозначать произведение $\left.m_{2} ! \cdots m_{n} !\right)$ :

$$
\sum_{\lambda \vdash n, A(\lambda)=k} n^{c-1} \frac{1}{l(\lambda)} \frac{l(\lambda) !}{m_{1} !|\operatorname{Aut}(\nu)|}=\sum_{\nu \vdash k} n^{c-1} \frac{1}{l(\lambda)} \frac{l(\lambda) !}{m_{1} !|\operatorname{Aut}(\nu)|} .
$$

Заметим, что

$$
A(\lambda)=\sum_{i=1}^{n}(i-1) m_{i}=\sum_{i=1}^{n} i m_{i}-\sum_{i=1}^{n} m_{i}=n-l(\lambda)
$$

и

$$
m_{1}=n-k-l(\nu) .
$$

Поэтому выражение (7) можно переписать в следующем виде:

$$
n^{c-1} \sum_{\nu \vdash k} \frac{1}{(n-k)|\operatorname{Aut}(\nu)|} \frac{(n-k) !}{(n-k-l(\nu)) !} .
$$

Докажем, что

$$
\sum_{\nu \vdash k} \frac{1}{(n-k)|\operatorname{Aut}(\nu)|} \frac{(n-k) !}{(n-k-l(\nu)) !}=\frac{1}{n}\left(\begin{array}{l}
n \\
k
\end{array}\right) .
$$

Перепишем равенство (8) в виде

$$
\sum_{\nu \vdash k} \frac{1}{|\operatorname{Aut}(\nu)|} \frac{(n-k) !}{(n-k-l(\nu)) !}=\left(\begin{array}{c}
n-1 \\
k
\end{array}\right) .
$$


Преобразуем левую часть равенства (9):

$$
\begin{aligned}
\sum_{\nu \vdash k} \frac{1}{|\operatorname{Aut}(\nu)|} \frac{(n-k) !}{(n-k-l(\nu)) !} & =\sum_{\nu \vdash k} \frac{l(\nu) !}{|\operatorname{Aut}(\nu)|} \frac{(n-k) !}{l(\nu) !(n-k-l(\nu)) !} \\
& =\sum_{\lambda \vdash k} \frac{l(\nu) !}{|\operatorname{Aut}(\nu)|}\left(\begin{array}{c}
n-k \\
l(\nu)
\end{array}\right) .
\end{aligned}
$$

Перегруппируем слагаемые в последней сумме формулы (10) по длине разбиения $\nu$ :

$$
\sum_{l=1}^{k}\left(\begin{array}{c}
n-k \\
l
\end{array}\right) \sum_{\nu \vdash k, l(\nu)=l} \frac{l !}{|\operatorname{Aut}(\nu)|}
$$

Заметим, что каждому неупорядоченному разбиению числа $k$ на $l$ слагаемых соответствует $l ! /|\operatorname{Aut}(\nu)|$ упорядоченных, а всего неупорядоченных разбиений имеется $\left(\begin{array}{c}k-1 \\ l-1\end{array}\right)$. Поэтому выражение (11) равно

$$
\sum_{l=1}^{k}\left(\begin{array}{c}
n-k \\
l
\end{array}\right)\left(\begin{array}{l}
k-1 \\
l-1
\end{array}\right)
$$

Нетрудно видеть, что выражение (12) равно $\left(\begin{array}{c}n-1 \\ k\end{array}\right)$. Действительно, $\left(\begin{array}{c}n-1 \\ k\end{array}\right)$ - это коэффициент при $x^{k}$ в многочлене $(1+x)^{n-1}$, а сумма $(12)$ - это коэффициент при $x^{k}$ в произведении двух многочленов $(1+x)^{n-k} \cdot(1+x)^{k-1}=(1+x)^{n-1}$.

Равенство (8), а вместе с ним и утверждение 2.1 доказано.

2. Доказательство леммы 1.3. Нетрудно заметить, что для доказательства леммы 1.3 достаточно просуммировать числа $N\left(\lambda_{1}, \ldots, \lambda_{c}\right)$ по всем наборам разбиений $\left\{\lambda_{1}, \ldots, \lambda_{c}\right\}$ вырожденностей $\left\{k_{1}, \ldots, k_{c}\right\}$. Выполним суммирование, воспользовавшись утверждением 2.1:

$$
\begin{aligned}
\operatorname{deg}_{k_{1}, \ldots, k_{c}} & =\sum_{\substack{\left\{\lambda_{1}, \ldots, \lambda_{c}\right\} \\
A\left(\lambda_{i}\right)=k_{i}}} N\left(\lambda_{1}, \ldots, \lambda_{c}\right) \\
& =\sum_{\substack{\left\{\lambda_{1}, \ldots, \lambda_{c}\right\} \\
A\left(\lambda_{i}\right)=k_{i}}} n^{c-1} \prod_{i=1}^{c} \frac{1}{l\left(\lambda_{i}\right)} \frac{l\left(\lambda_{i}\right) !}{m_{i 1} ! \cdots m_{i n} !} \\
& =n^{c-1} \prod_{i=1}^{c} \sum_{\substack{\lambda_{i} \\
A\left(\lambda_{i}\right)=k_{i}}} \frac{1}{l\left(\lambda_{i}\right)} \frac{l\left(\lambda_{i}\right) !}{m_{i 1} ! \cdots m_{i n} !}=n^{c-1} \prod_{i=1}^{c} \frac{1}{n}\left(\begin{array}{l}
n \\
k_{i}
\end{array}\right)=\frac{1}{n} \prod_{i=1}^{c}\left(\begin{array}{l}
n \\
k_{i}
\end{array}\right) .
\end{aligned}
$$

Последнее выражение в точности совпадает с требуемым в утверждении леммы 1.3. Доказательство завершено.

3. Доказательство теоремы 1.4. Напомним, что через $b_{n}(c)$ мы обозначали количество разложений полного цикла из $S_{n}$ в произведение $c$ перестановок, некоторые из которых могут быть тождественными. Числа $b_{n}(c)$ выражаются через вычисленные числа $\operatorname{deg}_{k_{1}, \ldots, k_{c}}$ следующим образом:

$$
b_{n}(c)=\sum_{k_{1}, \ldots, k_{c}, k_{1}+\cdots+k_{c}=n-1} \operatorname{deg}_{k_{1}, \ldots, k_{c}},
$$


где сумма берется по всем неупорядоченным наборам $k_{1}, \ldots, k_{c}$.

Для завершения доказательства теоремы 1.4 нужно доказать, что

$$
\sum_{k_{1}, \ldots, k_{c}, k_{1}+\cdots+k_{c}=n-1} \frac{1}{n}\left(\begin{array}{c}
n \\
k_{1}
\end{array}\right) \cdots\left(\begin{array}{c}
n \\
k_{c}
\end{array}\right)=\frac{1}{n}\left(\begin{array}{c}
c n \\
n-1
\end{array}\right) .
$$

Домножим обе части этого равенства на $n$. Справа стоит количество способов выбрать $n-1$ элементов из $c n$ элементов. Заметим, что сумма слева в точности равна этому количеству, так как рассматриваются всевозможные наборы $k_{1}, \ldots, k_{c}$ и всевозможные способы их выбора, т. е. каждый раз выбирается ровно $n-1$ элементов из $\mathrm{cn}$. Теорема доказана.

\section{ЛитерАтУРА}

[1] С. К. Ландо, Д. Звонкин, О кратностях отображения Ляшко-Лойенги на стратах дискриминанта, Функц. анализ и его прил., 33:3 (1999), 21-34.

[2] M. Bousquet-Melou, G. Schaeffer, Enumeration of planar constellations, Adv. Appl. Math., 24:4 (2000), 337-368.

[3] T. Ekedahl, S. K. Lando, M. Shapiro, A. Vainshtein, Hurwitz numbers and intersections on moduli spaces of curves, Invent. Math., 146 (2001), 297-327.

[4] I. P. Goulden, D. M. Jackson, The combinatorial relationship between trees, cacti and certain connection coefficients for the symmetric group, European J. of Combin., 13:5 (1992), 357-365.

[5] I. P. Goulden, D. M. Jackson, The KP-hierarchy, branched covers and triangulations, Adv. Math., 219:3 (2008), 932-951.

[6] A. Hurwitz, Über Riemann'sche Flächen mit gegebenen Verzweigungspunkten, Math. Ann., 39:1 (1891), 1-61.

[7] P. Johnson, Double Hurwitz numbers via the infinite wedge, http://arxiv.org/abs/ 1008.3266.

[8] E. Looijenga, The complement of the bifurcation variety of a simple singularity, Invent. Math., 23 (1974), 105-116.

[9] S. Shadrin, L. Spitz, D. Zvonkine, On double Hurwitz numbers with completed cycles, J. London Math. Soc., 86:2 (2012), 407-432.

Национальный исследовательский университет «Высшая школа экономики», Международная лаборатория математической физики и теории представлений e-mail: boris.bychkov@gmail.com 